\title{
An Update on Genetic Analysis of Cholestatic Liver Diseases: Digging Deeper
}

\author{
Roman Müllenbach Frank Lammert \\ Department of Medicine II, Saarland University Hospital, Homburg, Germany
}

\section{Key Words}

ABC transporters $\cdot$ Genome-wide scan $\cdot$ Personalized medicine $\cdot$ Polygenic disease

\begin{abstract}
Investigations into the molecular mechanisms of cholestasis have revealed intricate and intriguing details of bile salt metabolism as well as its regulatory mechanisms in health and disease. Extensive studies on genotype-phenotype correlations in monogenic diseases, such as progressive familial and benign recurrent intrahepatic cholestasis, facilitate diagnostics and improve the risk assessment of hepatobiliary transporter gene variants in bile transport pathophysiology. While the comparatively easy targets in monogenic cholestasis have been identified for some time now, progress in complex liver disease is rather laborious but steady. Genome-wide association scans are the next step in gathering information about common contributors towards polygenic (multifactorial) cholestatic diseases. New determinants of bile salt metabolism affecting feedback loops within the liver or the enterohepatic circulation are presently under investigation for their contribution towards complex cholestatic syndromes.

Copyright $\odot 2011$ S. Karger AG, Basel
\end{abstract}

(๑) 2011 S. Karger AG, Basel

0257-2753/11/0291-0072\$38.00/0

Accessible online at: www.karger.com/ddi

\section{Introduction}

Genomic information, i.e. the exact knowledge of an individual's genome, is becoming more accessible to the public [1]. This development - highlighted by the term 'consumer genomics' - is caused by the rapid decrease in cost and physical effort required to gather this information [2]. Accordingly, the speed of data generation is exceeding the level of understanding at an ever-increasing pace. To this end, the two major tasks at hand are: (i) communicating scientific advances in a manner that increases knowledge and awareness of the general population to allow a more informed choice of information available, and (ii) 'digging deeper' in analyzing the impact of gene variation on cells, organs and the whole human being in order to improve interpretation and prediction [3]. Several types of genetic testing are either in practice or envisioned for clinical use currently the most common being:

(1) Diagnostic tests for monogenic disease in symptomatic patients: Such tests are used in clinical practice, and we shall give some examples of how it has enhanced diagnosis. Knowledge from these so-called 'simple' diseases also provides good examples to illustrate the complexity of genetic diagnosis, such as gene $\times$ gene interaction ('epistasis') and incomplete penetrance.

Prof. Dr. Frank Lammert, MD

Department of Medicine II, Saarland University Hospital

Kirrberger Strasse

DE-66424 Homburg (Germany)

Tel.+496841 1623 201, E-Mail frank.lammert@uks.eu 
(2) Screening for monogenic hereditary diseases in asymptomatic individuals: These analyses can be population-based or targeted in families or groups with a known risk of being carriers of certain disease-associated variants. The results might be associated with a higher level of uncertainty than diagnostic testing in affected individuals [4]. Thus, they illustrate the difficulties associated with the next step, prediction and risk assessment.

(3) Assessment of polygenic predisposition in complex diseases (genomic susceptibility tests): The aim of these predisposition tests is to provide an indication of the absolute lifetime risk and/or or relative risk of an individual developing a condition compared with the general population [4].

Several cholestatic syndromes are Mendelian traits. A search in the database 'Online Mendelian Inheritance in Man' (http://www.ncbi.nlm.nih.gov/omim) for 'cholestasis' resulted in 88 hits. Most of these syndromes are amenable to genetic analysis to confirm the causative variant. The identification of an affected patient as carrying the respective mutant allows treatment to be tailored towards known effects of the affected gene, possibly suggest lifestyle alterations to prevent predictable future progression and aggravation (as in hemochromatosis), or simply confirm clinical identification. Genetic testing may be helpful for the following cholestatic syndromes:

(1) Alagille syndrome (http://www.ncbi.nlm.nih.gov/ omim/118450) is confirmed by searching for functional variants in JAG1, a NOTCH ligand [5]. However, even in such a seemingly simple setting, the severity of the resulting phenotype can vary widely, even on an identical genetic background, highlighting the complexity of gene $x$ environment interactions. Kamath et al. [6] reported monozygotic twins with Alagille syndrome who were concordant for a mutation in JAG1, but discordant for a clinical phenotype. One twin had severe pulmonary atresia with mild liver involvement, whereas the other had tetralogy of Fallot and severe hepatic involvement that required a liver transplant.

(2) Arthrogryposis, renal dysfunction and cholestasis (ARC) syndrome (http://www.ncbi.nlm.nih.gov/omim/ 208085) is caused by mutations in the VPS33 gene, which has a role in vacuolar protein sorting and lysosomal function. Sequencing of the VPS33 gene has been suggested as a first-line diagnostic test in ARC, rather than biopsy [7]. Considering the risks associated with biopsies, genetic tests certainly pose a valid alternative once a sufficiently detailed description is available or a sufficiently broad but detailed diagnostic tool has been established. Mutations in the gene encoding VIPAR, which

Update on Genetic Analysis of

Cholestatic Liver Diseases interacts with VPS33, have been shown to cause ARC syndrome in a minority of patients (http://www.ncbi. nlm.nih.gov/omim/613401). Cullinane et al. [8] concluded that the VPS33B-VIPAR complex has diverse functions in the pathways regulating apical-basolateral polarity in the liver and kidney.

(3) Progressive familial intrahepatic cholestasis (PFIC) types $1-3$ are confirmed by detection of functional variants in the ATP8B1 (PFIC type 1), ABCB11 (PFIC type 2) and $\mathrm{ABCB} 3$ (PFIC type 3 ) genes.

\section{Genetic Analysis and Diagnostic Tests for PFIC}

The identification of the molecules causing the three PFIC subtypes has laid the foundations for further inquiries into the etiology of complex cholestatic diseases. Differential diagnosis, phenotypic heterogeneity and incomplete penetrance of these 'simple' monogenic traits provide examples for the genetic complexity of monofactorial diseases. It draws a realistic picture of the anticipated complexity that will have to be addressed when interpreting genomic sequence data for complex risk analysis and predictions. Severe functional variants (often called 'mutations') in conserved regions of three canalicular ATP-binding transmembrane transporters have been shown to cause the three known PFIC subtypes 1-3 (table 1) [9-13]. The fact that all three transporters are located on the canalicular (basolateral) membrane indicates that this part of the hepatocyte is the 'bottleneck' of the enterohepatic circulation of bile acids: the least amount of functional redundancy, and the highest sensitivity to any disturbance.

(1) PFIC type 1 is caused by inactivation of the aminophospholipid-floppase (outside-in transporter) ATP8B1/ FIC-1 [10]. Functional impairment of this molecule is supposed to have an impact on all other basolateral transporter activities due to a disturbance in the membrane asymmetry, which is a requirement for transporter function [14], although the hepatic and extrahepatic phenotypes may be caused by other functions of this gene not yet identified [15]. In the lung, for example, ATP8B1 internalizes mitochondrial-specific diphosphatidylglycerol (cardiolipin), which disrupts surfactant from extracellular fluid [16].

(2) PFIC type 2 is caused by inactivation of the central and exclusive canalicular bile salt export pump ABCB11/ BSEP [11]. Bile salts are no longer transported into the bile canaliculus, causing chronic hepatocellular damage.

(3) PFIC type 3 results from sequence aberrations in conserved domains of the phospholipid-flippase (inside- 
Table 1. Genetic and biochemical characteristics of PFIC types 1-3 and the associated clinical phenotypes

\begin{tabular}{|c|c|c|c|}
\hline Type & 1 & 2 & 3 \\
\hline Gene & ATP8B1 (FIC1) & $A B C B 11$ (BSEP) & ABCB4 (MDR3) \\
\hline Phenotypes & $\begin{array}{l}\text { biliary cirrhosis } \\
\text { BRIC type } 1 \\
\text { extrahepatic manifestations: } \\
\text { malabsorption, pancreatitis, } \\
\text { deafness, pneumonia }\end{array}$ & $\begin{array}{l}\text { neonatal giant cell hepatitis } \\
\text { biliary cirrhosis } \\
\text { BRIC type } 2 \\
\text { gallstones } \\
\text { ICP } \\
\text { drug-induced cholestasis } \\
\text { HCC }\end{array}$ & $\begin{array}{l}\text { biliary cirrhosis with ductular } \\
\quad \text { proliferation } \\
\text { ICP } \\
\text { gallstones } \\
\text { HCC } \\
\text { CCA }\end{array}$ \\
\hline$\gamma-\mathrm{GT}$ & low! & low! & $\uparrow$ \\
\hline Therapy & $\begin{array}{l}\text { biliary diversion } \\
\text { OLT }\end{array}$ & $\begin{array}{l}\text { biliary diversion } \\
\text { OLT }\end{array}$ & $\begin{array}{l}\text { ursodeoxycholic acid } \\
\text { OLT }\end{array}$ \\
\hline
\end{tabular}

BRIC = Benign recurrent intrahepatic cholestasis; $\mathrm{CCA}=$ cholangiocarcinoma; HCC = hepatocellular carcinoma; ICP = intrahepatic cholestasis of pregnancy; OLT = orthotopic liver transplantation.

out transporter) ABCB4/MDR3 [17]. Phospholipids, in concert with cholesterol, aid in protecting the bile canaliculi from the aggressive detergent action of bile salts by forming mixed micelles, the normal bile. Lack of ABCB4 activity results in aggressive 'toxic' bile, which destroys the bile ducts.

In these high-penetrance monogenic cholestatic diseases, sequence analysis of the complete gene, comprising the coding region, flanking splice sites and possibly the regulatory region, has been recommended. Simple clinical chemical tests may help to decide which transporter gene to start sequencing with. Whereas PFIC3, caused by $A B C B 4$ mutations, is usually distinguishable from type 1 and 2 by increased $\gamma$-GT values due to the toxic action of nonmicellar bile acids, differential diagnosis is more demanding when distinguishing between the two latter types. Pawlikowska et al. [18] and Davit-Spraul et al. [19] indicate that a predominant cholestatic pattern (AP/ ALT $\uparrow$ ) points to PFIC1, whereas elevated aminotransferase activities (ALT/AP $\uparrow$ ) indicate ABCB11 deficiency (PFIC2). Functional variants are highly heterogeneous and only in PFIC2, the majority of patients of European origin (58-61\%) carry one of the two more frequent founder mutations E297G and D482G [13]. In clinical practice, immunohistochemistry for transporters can also be very helpful to verify PFIC in individual cases, since complete absence of the respective protein is diagnostic.

\section{From Monogenic Disease to Polygenic Susceptibility}

In PFIC3, the observation that the mothers of children suffering from this disease had an increased likelihood for episodes of intrahepatic cholestasis of pregnancy (ICP) provided a first clue that some cases of ICP might be caused by variants in the selfsame gene(s). Accordingly, genetic variants causing severe functional impairment when present in homozygous form in PFIC3 children could be demonstrated in women with ICP $[12,20]$. Statistical analysis of $A B C B 4$ polymorphisms in women with ICP provided the first hints towards a role of seemingly 'normal' $A B C B 4$ genotypes towards predisposition, particularly a variant in exon $8[21,22]$. A more detailed genetic examinations of genotype and haplotype distributions among ICP patients showed that the $[\mathrm{A}]$ allele at position 711 in exon 8 of the $A B C B 4$ gene conveys an increased risk of raised serum bile salt levels $(>40 \mu \mathrm{mol} / \mathrm{l})$ during pregnancy, one of the cutoff criteria for the severe subtype of ICP [23].

The spectrum of $A B C B 4$-associated diseases illustrates that severe functional variants causing congenital cholestatic diseases can be described as the 'tip of the iceberg' when it comes to the genetic background of cholestasis. They provide positional information on where to look for causative genes, and as such give us leverage to dissect other phenotypes that might relate or overlap with the wide spectrum of cholestatic syndromes. The genes 
identified in PFIC are 'beacons', and in ICP as well as in drug-induced cholestasis, the investigation of these genes has already provided a rich harvest of insight into the contribution and effect of less severe variants towards heterogeneous syndrome, including biliary fibrosis and cholelithiasis [4].

\section{From Genotype to Phenotype}

A comprehensive overview of genetic variants in patients with PFIC 2 described a total of 82 different $A B C B 11$ mutations in 109 families [13]. In an initial attempt to dissect the genotype to phenotype (G2P) challenge, Strautnieks and colleagues $[13,24]$ found that variants resulting in protein truncation carried the highest risk of developing cancer (hepatocellular carcinoma or cholangiocarcinoma), whereas some missense mutations still showed residual ABCB11 immunostaining. Byrne et al. [25] and Ho et al. [26] beautifully illustrated the wide spectrum of functional abnormalities, which ranged from severe truncation by a premature stop codon resulting in total absence of ABCB11 protein to preferential exon splicing or skipping [27]. Partial degradation or retention of misfolded protein in the endoplasmic reticulum and nonsensemediated RNA decay are mechanisms inside the cell that reduce the amount of protein available at the basolateral membrane. The possibility to 'release' misfolded protein from retention in the endoplasmic reticulum by treatment with 4-phenylbutyrate has been considered and shown to ameliorate ABCB11 deficiency in vitro [28, 29].

\section{The Common $A B C B 11$ Risk Variant}

The potential role of frequent gene variants in disease predisposition is illustrated by the p.A444V polymorphism in the bile salt export pump (fig. 1). The presence of the [C] allele at position 1,331 of the cDNA (resulting in alanine at position 444 in the protein) has been associated with lower hepatic expression of the protein [30]. Genotyping of this variant in patients suffering from various forms of cholestasis has revealed a significantly skewed allele distribution, with the [C] allele conferring increased risk in drug-induced cholestasis [31] as well as contraceptive-induced cholestasis and cholestasis of pregnancy [32, 33] (fig. 1). It would certainly be interesting to study a sufficiently large number of PFIC1 and PFIC3 patients for the impact of the common $A B C B 11$ p.A444V variant in modulating disease phenotypes.

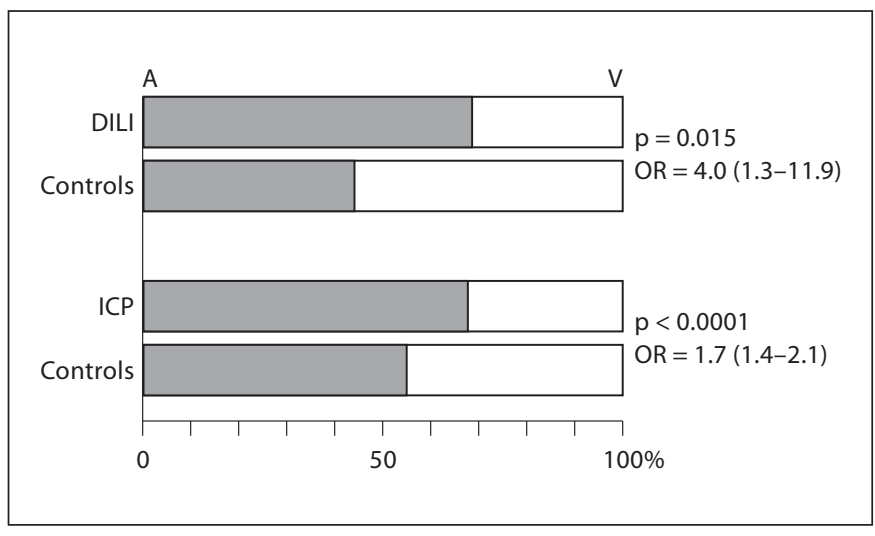

Fig. 1. $A B C B 11$ p.A444V allele distribution.

\section{Assessment of Polygenic Predisposition to Cholestatic Disease}

One of the initial aims of the human genome project, and here we return to the initial subject of 'consumer genetics', was to know the total sequence of the human genome and use it to elucidate the consequences of variation. The first goal has been accomplished, and due to the increasing speed of analysis and data processing, it is a question of years rather than decades as to when we can assess risk factors in an individual on the basis of a personal genome scan. One of the most successful 'workhorses' to identify new and unknown gene variants associated with disease progression and severity has been the genome-wide association scan (GWAS), where large sets of markers covering the entire genome are tested for association in populations and the matching controls $[4$, 34-36].

Statistical analysis, including correction for multiple testing, identifies loci where a difference of genotype distribution between cases and controls indicates the prevalence of a certain allele or genotype in one or the other population. While this is no proof of involvement (as often there is a lack of functional evidence for the contribution of the respective marker or nearby gene), these studies provide signaling peaks that indicate the presence of a contributory factor nearby. If the association is strong enough, markers from the respective region can be used as tools to quantify or stratify patients' risk without further need to identify the functional variant causing the observed effect.

The graphic presentations of the GWAS results, known as 'Manhattan plots', illustrate the 'shallow sweep' nature 
of this methodology [4]. The horizontal axis of a Manhattan plot shows the relative lengths of the chromosomes, and each dot of the plot represents a different single nucleotide polymorphism that was genotyped successfully in the study; the vertical axis shows the p values for each polymorphism.

Only loci conveying a certain (albeit low) level of risk in a relatively high proportion of patients result in a significant association peak in a GWAS. Due to the complex nature of factors involved, every single strategy to further dissect the loci has a preponderance to pick up certain types of candidate genes but miss out on others. Candidate gene sequencing and GWAS studies pick up the extreme ends of the spectrum: very low-frequency, highimpact variants in case of the former, juxtaposed by highfrequency, low-impact variants detected in the latter.

To assess gene interactions between variants that might be without any consequence when seen as single entities, but aggravate towards a clinical phenotype in combination (so-called 'epistatic interactions'), we need to apply additional strategies [4]. Finding patients that carry a combination of mildly detrimental variants in several known contributory genes and display a clinical phenotype allows us to retrospectively study the genotype-to-phenotype relationship in the patient and compare it to the (ideally unaffected) parents and siblings [37]. This type of 'retrospective family analysis' might provide us with clues on how the combination of risk alleles can aggravate nonsymptomatic alterations seen in unaffected relatives. Although this manner of gathering and combining data is tedious and fraught with problems of comparability and background heterogeneity, it can still provide good pointers as to the contribution of various loci once there are sufficient observations to reach significance level.

\section{From Animal Models to Human Disease}

While it is entirely logical and feasible to conceive interactions between the impact of various susceptibility genes, it is much harder to prove and quantify cumulative, additive or other epistatic effects. Animal models of complex diseases are being used to identify modifiers of severe functional variants, as demonstrated in the $A b c b 4$ knockout mouse [17] on different background strains. The phenotype of the Abcb4 knockout is different between different mouse strains, suggestive of a role for genetic modifiers. Crossing a fibrosis-susceptible strain with a resistant strain and phenotyping the intercross
(F2) offspring is a way of identifying genomic loci that confer increased or decreased susceptibility to cholestasis caused by ABCB4 deficiency.

Genome-wide linkage analysis ('quantitative trait locus mapping') of the trait 'fibrosis', defined by collagen accumulation and measured by hydroxyproline assay, has identified a significant modifier locus on mouse chromosome 12 and a suggestive locus on chromosome 4 [Hall R, Grünhage F, Lammert F; unpubl. observations]. Studying epistatic interactions in the F2 progeny revealed that a combination of inherited loci on mouse chromosomes 11 and 12 modifies the fibrotic phenotype considerably. The analysis of pairwise interactions confirmed chromosome 12 as a modifier locus for fibrosis in the $A b c b 4$ knockout mouse and showed further interaction with a locus on chromosome 9.

\section{Conclusions}

A genotype-to-phenotype correlation is never simple, not even in so-called 'simple' or monogenic Mendelian cholestatic diseases for which genetic tests do not deliver a clear-cut diagnosis. GWAS and complex mapping studies in inbred animals are tools to identify previously unsuspected modifier loci and, eventually, genes predisposing to cholestasis. The resulting risk matrices are bound to be of high complexity, and we have to keep on striving to convey an understanding of this complexity to our patients with cholestatic liver diseases and our peers.

\section{Disclosure Statement}

The authors declare that no financial or other conflict of interest exists in relation to the content of the article. R.M. and F.L. received funding from the German Research Council (DFG-LA 997/5-1)

\footnotetext{
References $\quad 1$ Getting personal. Nature 2008;455:1007.

2 European Society of Human Genetics: Statement of the ESHG on direct-to-consumer genetic testing for health-related purposes. Eur J Hum Genet 2010;18:1271-1273.

3 Positively disruptive. Nat Genet 2008;40:119.

4 Krawczyk M, Müllenbach R, Weber SN, et al: Genome-wide association studies and genetic risk assessment of liver diseases. Nat Rev Gastroenterol Hepatol 2010;7:669-681.

$\checkmark 5$ Oda T, Elkahloun AG, Pike BL, et al: Mutations in the human Jagged1 gene are responsible for Alagille syndrome. Nat Genet 1997; 16:235-242.
} 
6 Kamath BM, Krantz ID, Spinner NB, et al: Monozygotic twins with a severe form of Alagille syndrome and phenotypic discordance. Am J Med Genet 2002;112:194-197.

$\checkmark 7$ Gissen P, Tee L, Johnson CA, et al: Clinical and molecular genetic features of ARC syndrome. Hum Genet 2006;120:396-409.

$\checkmark 8$ Cullinane AR, Straatman-Iwanowska A, Zaucker A, et al: Mutations in VIPAR cause an arthrogryposis, renal dysfunction and cholestasis syndrome phenotype with defects in epithelial polarization. Nat Genet 2010;42:303-312.

-9 Deleuze JF, Jacquemin E, Dubuisson C, et al: Defect of multidrug-resistance 3 gene expression in a subtype of progressive familial intrahepatic cholestasis. Hepatology 1996; 23:904-908.

10 Bull LN, van Eijk MJ, Pawlikowska L, et al: A gene encoding a P-type ATPase mutated in two forms of hereditary cholestasis. Nat Genet 1998;18:219-224.

-11 Strautnieks SS, Bull LN, Knisely AS, et al: A gene encoding a liver-specific $A B C$ transporter is mutated in progressive familial intrahepatic cholestasis. Nat Genet 1998;20: 233-238.

12 Jacquemin E, De Vree JM, Cresteil D, et al: The wide spectrum of multidrug resistance 3 deficiency: from neonatal cholestasis to cirrhosis of adulthood. Gastroenterology 2001; $120: 1448-1458$

-13 Strautnieks SS, Byrne JA, Pawlikowska L, et al: Severe bile salt export pump deficiency: 82 different ABCB11 mutations in 109 families. Gastroenterology 2008;134:1203-1214.

- 14 Paulusma CC, Groen A, Kunne C, et al: Atp8b1 deficiency in mice reduces resistance of the canalicular membrane to hydrophobic bile salts and impairs bile salt transport. Hepatology 2006;44:195-204.

15 Verhulst PM, van der Velden LM, Oorschot $\mathrm{V}$, et al: A flippase-independent function of ATP8B1, the protein affected in familial intrahepatic cholestasis type 1 , is required for apical protein expression and microvillus formation in polarized epithelial cells. Hepatology 2010;51:2049-2060.

16 Ray NB, Durairaj L, Chen BB, et al: Dynamic regulation of cardiolipin by the lipid pump Atp8b1 determines the severity of lung injury in experimental pneumonia. Nat Med 2010;16:1120-1127.
17 Smit JJ, Schinkel AH, Oude Elferink RP, et al: Homozygous disruption of the murine $\mathrm{mdr} 2$ $\mathrm{P}$-glycoprotein gene leads to a complete absence of phospholipid from bile and to liver disease. Cell 1993;75:451-462.

18 Pawlikowska L, Strautnieks S, Jankowska I, et al: Differences in presentation and progression between severe FIC1 and BSEP deficiencies. J Hepatol 2010;53:170-178.

19 Davit-Spraul A, Fabre M, Branchereau S, et al: ATP8B1 and ABCB11 analysis in 62 children with normal gamma-glutamyl transferase progressive familial intrahepatic cholestasis (PFIC): phenotypic differences between PFIC1 and PFIC2 and natural history. Hepatology 2010;51:1645-1655.

20 Dixon PH, Weerasekera N, Linton KJ, et al: Heterozygous MDR3 missense mutation associated with intrahepatic cholestasis of pregnancy: evidence for a defect in protein trafficking. Hum Mol Genet 2000;9:12091217.

21 Müllenbach R, Linton KJ, Wiltshire S, et al: $\mathrm{ABCB} 4$ gene sequence variation in women with intrahepatic cholestasis of pregnancy. J Med Genet 2003;40:e70.

-22 Savander M, Ropponen A, Avela K, et al: Genetic evidence of heterogeneity in intrahepatic cholestasis of pregnancy. Gut 2003;52: 1025-1029.

23 Wasmuth HE, Glantz A, Keppeler H, et al: Intrahepatic cholestasis of pregnancy: the severe form is associated with common variants of the hepatobiliary phospholipid transporter ABCB4 gene. Gut 2007;56:265-270.

-24 Knisely AS, Strautnieks SS, Meier Y, et al: Hepatocellular carcinoma in ten children under five years of age with bile salt export pump deficiency. Hepatology 2006;44:478486.

25 Byrne JA, Strautnieks SS, Ihrke G, et al: Missense mutations and single nucleotide polymorphisms in ABCB11 impair bile salt export pump processing and function or disrupt pre-messenger RNA splicing. Hepatology 2009;49:553-567.

26 Ho RH, Leake BF, Kilkenny DM, et al: Polymorphic variants in the human bile salt export pump (BSEP; ABCB11): functional characterization and interindividual variability. Pharmacogenet Genomics 2010;20: 45-57.
27 Müllenbach R, Lammert F: The transporter 'variome': the missing link between gene variants and bile salt transporter function. Hepatology 2009;49:352-354.

28 Hayashi H, Sugiyama Y: 4-phenylbutyrate enhances the cell surface expression and the transport capacity of wild-type and mutated bile salt export pumps. Hepatology 2007;45: 1506-1516.

29 Van der Velden LM, Stapelbroek JM, Krieger E, et al: Folding defects in P-type ATP 8B1 associated with hereditary cholestasis are ameliorated by 4-phenylbutyrate. Hepatology 2010;51:286-296.

-30 Meier Y, Pauli-Magnus C, Zanger UM, et al: Interindividual variability of canalicular ATP-binding-cassette (ABC)-transporter expression in human liver. Hepatology 2006; 44:62-74.

31 Lang C, Meier Y, Stieger B, et al: Mutations and polymorphisms in the bile salt export pump and the multidrug resistance protein 3 associated with drug-induced liver injury. Pharmacogenet Genomics 2007; 17: 47-60.

32 Meier Y, Zodan T, Lang C, et al: Increased susceptibility for intrahepatic cholestasis of pregnancy and contraceptive-induced cholestasis in carriers of the 1331T $>$ C polymorphism in the bile salt export pump. World J Gastroenterol 2008;14:38-45.

33 Dixon PH, van Mil SW, Chambers J, et al: Contribution of variant alleles of ABCB11 to susceptibility to intrahepatic cholestasis of pregnancy. Gut 2009;58:537-544.

34 Hirschfield GM, Liu X, Xu C, et al: Primary biliary cirrhosis associated with HLA, IL12A, and IL12RB2 variants. N Engl J Med 2009;360:2544-2555.

- 35 Daly AK, Donaldson PT, Bhatnagar P, et al: HLA-B ${ }^{*} 5701$ genotype is a major determinant of drug-induced liver injury due to flucloxacillin. Nat Genet 2009;41:816-819.

36 Liu X, Invernizzi P, Lu Y, et al: Genome-wide meta-analyses identify three loci associated with primary biliary cirrhosis. Nat Genet 2010;42:658-660.

37 Zimmer V, Müllenbach R, Simon E, et al: Combined functional variants of hepatobiliary transporters and FXR aggravate intrahepatic cholestasis of pregnancy. Liver Int 2009;29:1286-1288. 